\title{
LSST Primary/Tertiary Monolithic Mirror
}

\author{
J. Sebag ${ }^{1}$, W. Gressler ${ }^{1}$, M. Liang ${ }^{2}$, D. Neill ${ }^{1}$, C. Araujo-Hauck ${ }^{1}$, J. Andrew ${ }^{1}$, G. Angeli ${ }^{1}$, M. Cho $^{1}$, C.

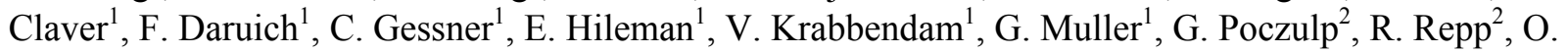 \\ Wiecha $^{1}$, B. Xin ${ }^{1}$, K. Kenagy ${ }^{3}$, H. M. Martin ${ }^{3}$, M. T. Tuell ${ }^{3}$, S. C. West ${ }^{3}$ \\ ${ }^{1}$ Large Synoptic Survey Telescope, 950 N Cherry Ave, Tucson, AZ, 85719, USA \\ ${ }^{2}$ National Optical Astronomy Observatory, 950 N Cherry Ave, Tucson, AZ 85719, USA \\ ${ }^{3}$ Steward Observatory, the University of Arizona, Tucson, AZ 85721, USA
}

\begin{abstract}
At the core of the Large Synoptic Survey Telescope (LSST) three-mirror optical design is the primary/tertiary (M1M3) mirror that combines these two large mirrors onto one monolithic substrate. The M1M3 mirror was spin cast and polished at the Steward Observatory Mirror Lab at The University of Arizona (formerly SOML, now the Richard F. Caris Mirror Lab at the University of Arizona (RFCML)). Final acceptance of the mirror occurred during the year 2015 and the mirror is now in storage while the mirror cell assembly is being fabricated. The M1M3 mirror will be tested at RFCML after integration with its mirror cell before being shipped to Chile.
\end{abstract}

Keywords: LSST, Mirror, M1M3, Structure Function, Transportation, Crow's Feet

\section{INTRODUCTION}

The Large Synoptic Survey Telescope (LSST) Project is a public-private partnership to conduct a wide, fast, deep survey and to process and serve the data ${ }^{1}$. This mission is achieved via a three-mirror wide field of view optical design, a 3.2-Gpixel camera ${ }^{2}$, and an automated data processing system. At the core of the three-mirror optical design is the M1M3 mirror that combines the primary mirror and the tertiary mirror onto one monolithic substrate ${ }^{3-6}$. This uncommon configuration enabled the design of a compact and stiff telescope mount structure to meet the slew and settle specifications required for the LSST survey ${ }^{7}$.

This paper summarizes the results from the M1M3 mirror final acceptance. All critical parameters were measured with sufficient accuracy to demonstrate compliance with the specifications ${ }^{8}$. For each mirror M1 and M3, the vertex radius of curvature, conic constant, and position of the optical axis with respect to the mechanical axis of the blank were demonstrated to be within the specifications including the relative position of M3 optical axis relative to M1 optical axis. The results from the optical design optimization using the as-built measured values for these parameters is presented.

This paper also details the holes and the "crow's feet" flaws that affect the mirror surface. Both of these defects are linked with each other. The results of the testing done to fill the holes are discussed. The crow's feet high resolution images measured on the mirror are presented here. Their impact on the LSST image quality are discussed elsewhere ${ }^{8,9}$.

Ground-based and Airborne Telescopes VI, edited by Helen J. Hall, Roberto Gilmozzi, Heather K. Marshall, Proc. of SPIE Vol. 9906, 99063E · C 2016 SPIE · CCC code: 0277-786X/16/\$18 · doi: 10.1117/12.2230012 
This paper includes a discussion of the M1M3 finite element model (FEM). This model was updated to match the as-built mirror by incorporating all thickness measurements and mirror support definition. Finally, the transportation of the M1M3 mirror to a secure facility for storage is described. It happened toward the middle of May 2015 after the LSST Project took responsibility for the mirror in its shipping container. The mirror is expected to remain in storage for approximately two years until the operational M1M3 mirror cell assembly is fabricated, at which time the M1M3 mirror and mirror cell can be integrated and tested before being shipped to Chile.

\section{M1M3 FINAL ACCEPTANCE}

The fabrication and testing of the M1M3 mirror was finished in October 2014 and was followed by an acceptance testing period of 4 months (until February 2015) in order to perform all the required verifications for this uncommon design. Working closely with the RFCML team during the last year of fabrication allowed the LSST project to develop its own processing pipeline for the interferometric data. This effort was critical to facilitate the acceptance process by enabling easy communication between both teams and better understanding of this intricate data processing.

Table 1: Required vs measured M1M3 prescription

\begin{tabular}{|l|c|c|}
\hline M1M3 Parameters & Required & Measured \\
\hline Outside Diameter & $8417 \pm 1 \mathrm{~mm}$ & $8417.5 \pm 0.2 \mathrm{~mm}$ \\
\hline Inside diameter & $1054.7+2 /-0 \mathrm{~mm}$ & $1054.8 \pm 0.1 \mathrm{~mm}$ \\
\hline Substrate thickness at r=4208.5 mm & $919 \pm 2 \mathrm{~mm}$ & $919.5 \pm 0.2 \mathrm{~mm}$ \\
\hline Mean facesheet thickness & $28 \pm 1 \mathrm{~mm}$ & $28.4 \pm 0.2 \mathrm{~mm}$ \\
\hline M1 optical axis to substrate mech. axis (radial) & $<1 \mathrm{~mm}$ & $0.3 \pm 0.3 \mathrm{~mm}$ \\
\hline M3 optical axis to M1 optical axis (radial) & $<1 \mathrm{~mm}$ & $0.4 \pm 0.4 \mathrm{~mm}$ \\
\hline M3 vertex height below M1 vertex & $233.8 \pm 2 \mathrm{~mm}$ & $234.4 \pm 0.1 \mathrm{~mm}$ \\
\hline M3 Wedge & $0<100 \mu \mathrm{m} \mathrm{TIR}$ & $16 \pm 20 \mu \mathrm{m} \mathrm{TIR}$ \\
\hline Microroughness & $0<20 \AA \mathrm{rms}$ & $15 \pm 3 \AA \mathrm{rms}$ \\
\hline M1 vertex radius & $19835.5 \pm 1 \mathrm{~mm}$ & $19835.1 \pm 0.2 \mathrm{~mm}$ \\
\hline M3 vertex radius & $8344.7 \pm 1 \mathrm{~mm}$ & $8344.1 \pm 0.1 \mathrm{~mm}$ \\
\hline M1 conic constant $(\mathrm{k})$ & $-1.2150 \pm 0.0002$ & $-1.21502 \pm 0.00011$ \\
\hline M3 conic constant $(\mathrm{k})$ & $+0.1550 \pm 0.0001$ & $+0.15497 \pm 0.00005$ \\
\hline
\end{tabular}

Optical surfaces and their alignment were measured by a combination of interferometric tests and laser tracker measurements (table1). The final acceptance interferometric data was acquired in October 2014 and December 2014 (figure 1). Vignetting from the M3 bridge is visible on the December results because the interferometric data was obtained simultaneously on both mirrors while a sequence of measurements taken successively on M1, then M3 and then M1 was used in October. Additional measurements were taken after rotating the M1M3 mirror 180deg.

The structure functions (SF) computed on all the different interferometric data sets met the specifications (figure 2). The M1 SF was well below the specification for all test configurations except around a separation close to $0.1 \mathrm{~m}$ where it was practically tangent to the specification. The M3 SF was slightly more variable with the test configuration. Some residual low frequency bending modes present for the M1M3 simultaneous measurement configuration raised the SF toward the specification for separation above $1 \mathrm{~m}$. In addition, the M3 SF was also practically tangent to the specification for separation around $0.06 \mathrm{~m}$. 


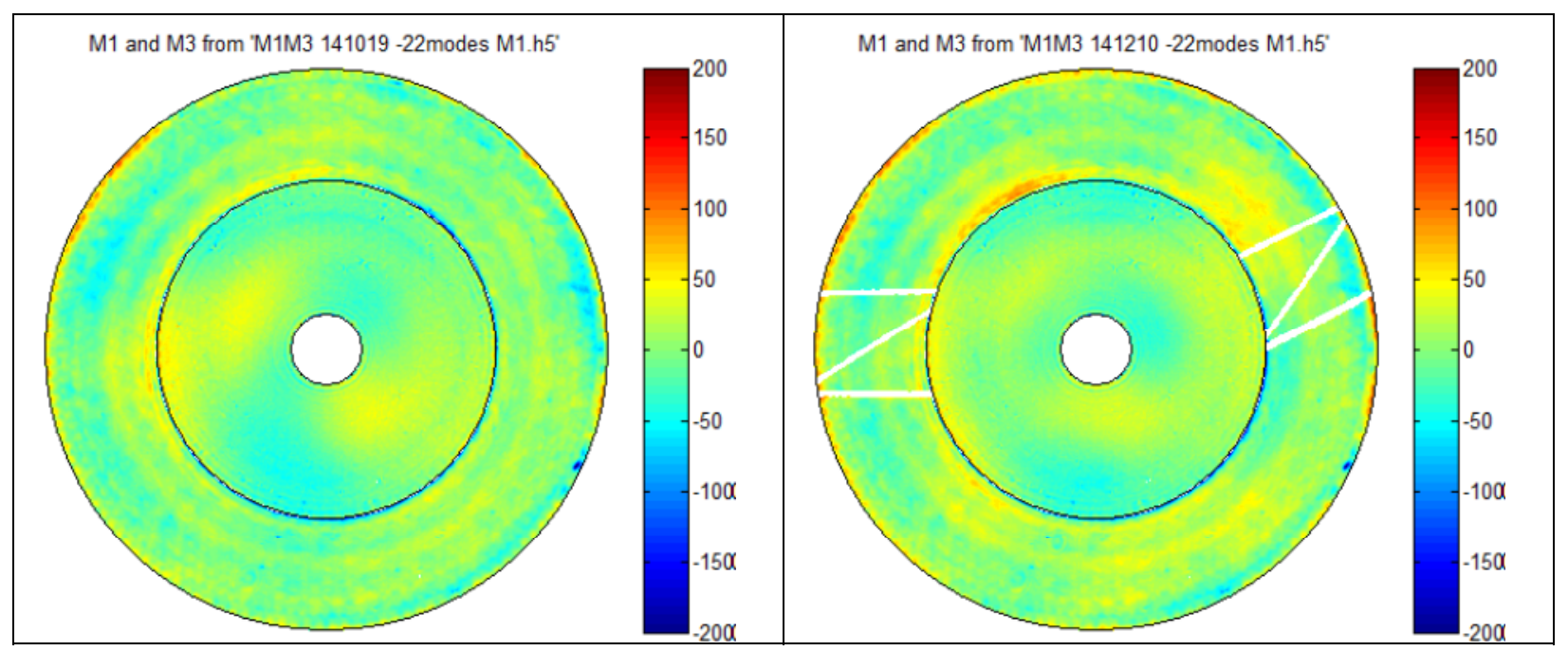

Figure 1: M1M3 mirror surface after processing of acceptance interferometric data acquired on October 192014 (left) and December 102014 (right). The vignetting visible over the M1 mirror (right) is due to the bridge holding the M3 interferometer during simultaneous measurements. It is not visible on the M1 mirror (left) during the sequence Mlonly, M3 only, M1 only because the bridge is retracted during the MI measurement.

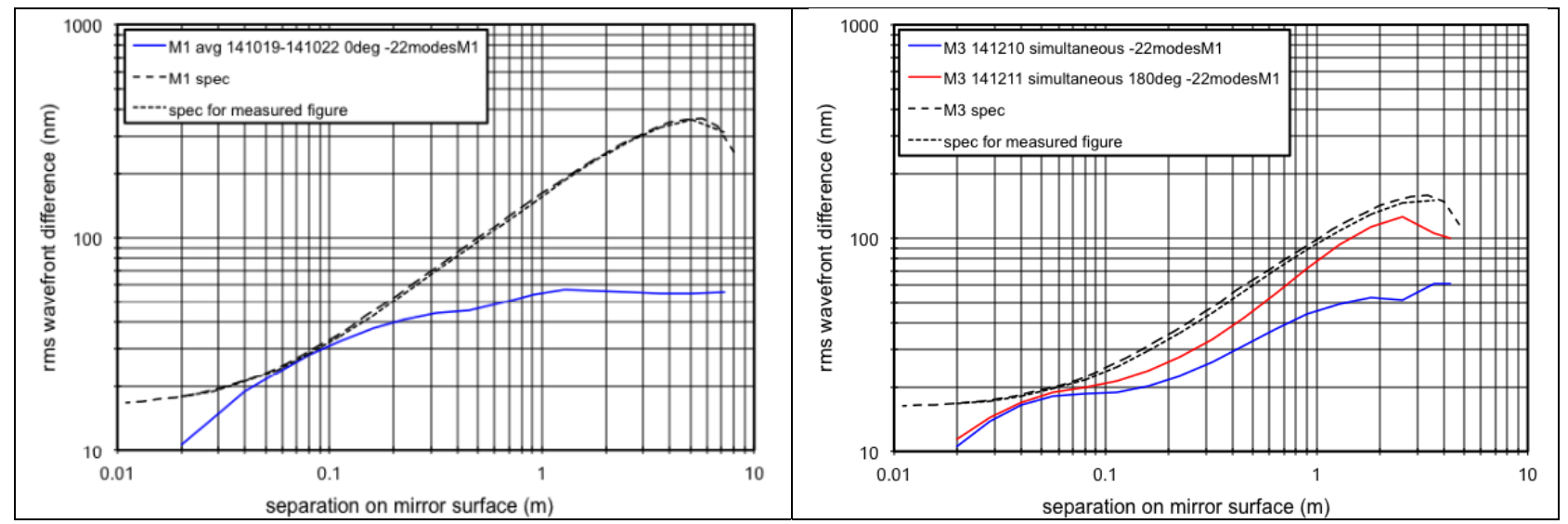

Figure 2: M1 structure function vs specification (left) and M3 structure function vs specification (right). The dotted line is the specification corrected for the measurement error.

This M1 SF was computed from the M1 mirror surface measurement averaged over all the acceptance data from October 19 to October 22 after processing and subtraction of the first 22 bending modes. Two M3 SF are plotted from the M3 mirror surface simultaneous measurements at 0deg and 180deg acquired on December 10 and 11, 2014 after processing and subtraction of the first 22 bending modes.

A large part of the acceptance tests was dedicating to image quality impact estimation of mirror surface defects called the "crow's feet". These were found in large number on the M1M3 surface. Their origin is linked to the interaction between the polishing compound and the sharp edge of small open bubbles on the mirror surface. Both of these topics are described in more details in the next paragraphs.

Finally, the positions of the spherically mounted retroreflectors (SMR) bonded to the mirror outer sidewall (figure 3) were measured relative to the M1 optical axis using a laser tracker. These will be used 
during operations to align the M2 mirror and the camera to the M1 optical axis. The method uses the bestfit circle of the 12 SMRs to define the $z$ axis and the $x-y$ plane of the SMR referential. SMR \#11 defines the $+x$ direction. Using this coordinate system, the coordinates of two points on the optical axis were identified: the M1 vertex and the M1 center of curvature (table 2).

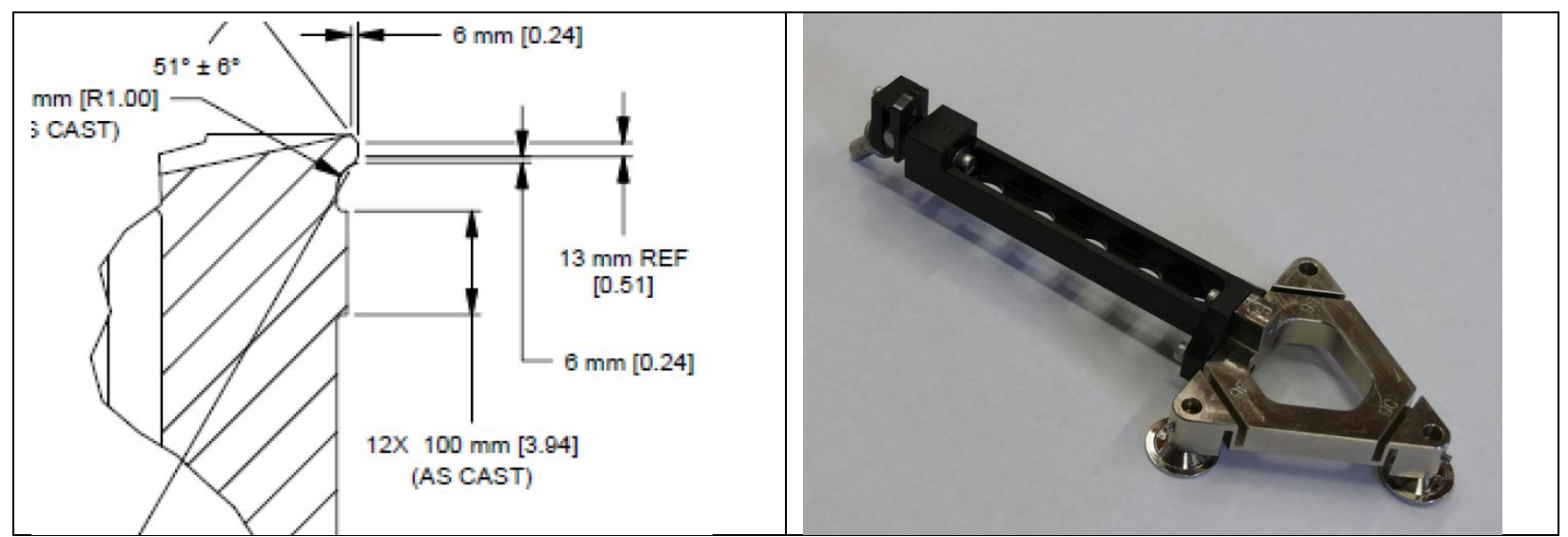

Figure 3: M1 mirror outer sidewall showing the boss where the support tower for the SMR is bonded (left). These were cast during fabrication of the mirror. One of the 12 SMR support towers (right) before bonding of the triangular support on the mirror sidewall.

Table 2: Coordinates of the M1 vertex and center of curvature relative to the referential defined by the SMR located around the M1 perimeter. Values are in $\mathrm{mm}$.

\begin{tabular}{|l|c|c|c|}
\hline \multicolumn{1}{|c|}{ M1 Optical Axis } & $\mathrm{x}$ & $\mathrm{y}$ & $\mathrm{z}$ \\
\hline M1 Vertex position & 0.229 & -0.223 & -460.161 \\
\hline M1 Center of Curvature & -0.213 & 2.396 & 19375.340 \\
\hline
\end{tabular}

\section{M1M3 BUBBLES AND HOLES}

A large amount of small air bubbles are confined within the glass used to manufacture the M1M3 mirror. During the mirror fabrication, many of these bubbles become exposed at the mirror surface. They are usually very small and very difficult to locate visually on the mirror surface. They form a tiny circular hole on the mirror surface. The hole with the largest diameter size found on the LSST M1M3 mirror surface is equal to $2.5 \mathrm{~mm}$. Such large holes are usually detected and their edge treated during fabrication. However, after careful inspection, a large amount of less than $1 \mathrm{~mm}$ diameter holes was also found on the M1M3 mirror. Estimates based on a survey of 54 cores from center to edge result in around 6800 bubbles with diameter $\geq 0.2 \mathrm{~mm}$ on the whole surface.

The presence of these holes has a potential impact on the mirror cleaning process quality executed before coating the mirror. LSST is developing a coating plant ${ }^{10}$ that will include an air knife to dry the mirror. The air knife dries the mirror by blowing filtered dry air on the mirror surface to direct any liquid toward the mirror center hole where it is drained. The concern is that liquid could get trapped inside the holes and leave some residue that would eventually reduce the coating lifetime.

One possible solution would be to fill the holes with an appropriate UV curing cement to avoid this situation. To test this idea, nine holes of varying sizes were drilled into each of two 2"x2" BK7 and Pyrex coated witness samples to determine if the liquids used for stripping and cleaning the surface prior to coating would contaminate it during the drying process. UV curing cement was applied to the nine holes 
in the first sample (B06) and to six of the nine holes in the second sample (P37) to fill the holes and prevent them from becoming wells of liquid that would spread around during compressed air drying (simulating an air knife). Norland Optical Adhesive (NOA) 61 was used on sample B06. One column was filed with the same cement on sample P37 but one other column was filled with NOA 71 instead for comparison. Sample B06 was also cold cycled several times and tested interferometrically to determine the figure stability after filling the nine holes with this product (figure 4). No mirror figure impact was detected after the cold cycles.

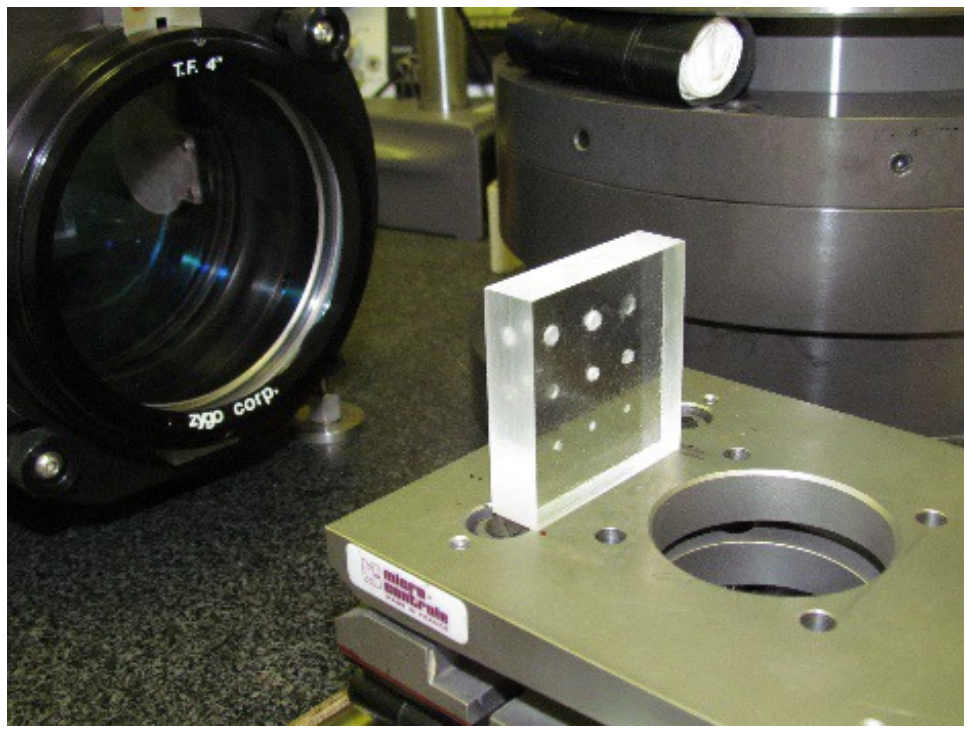

Figure 4: Sample B06 during interferometric

Then, both samples (B06 and P37) were stripped of aluminum and cleaned per standard large mirror cleaning practices - with one exception. Instead of drying the sample by wiping after the final rinse, it was blown dry with N2 to simulate the effect of drying a large surface with an air knife. Both samples were then sputter coated with protected silver at the Gemini South magnetron equipped chamber.

Of the 18 holes in the two substrates (15 filled with UV curing cement, 3 unfilled) only one hole showed surface contamination due to residual moisture trapped during the blow-off drying (figure 5). Unexpectedly, it was one of the larger holes on B06 that had all of its holes filled with UV curing cement. There appears to be a very small gap between the UV curing cement and the edge of the hole that trapped a small amount of residual moisture. Examination after drying prior to coating showed no indication of the spray pattern, it was only visible after the sample had been coated. In addition, the unfilled holes did not show any trace of contamination as was originally feared. The adhesion test performed in the contaminated area show poor adhesion results (figure 6). Based on these results, the conclusion was to keep the mirror surface as is and to not fill the holes. 


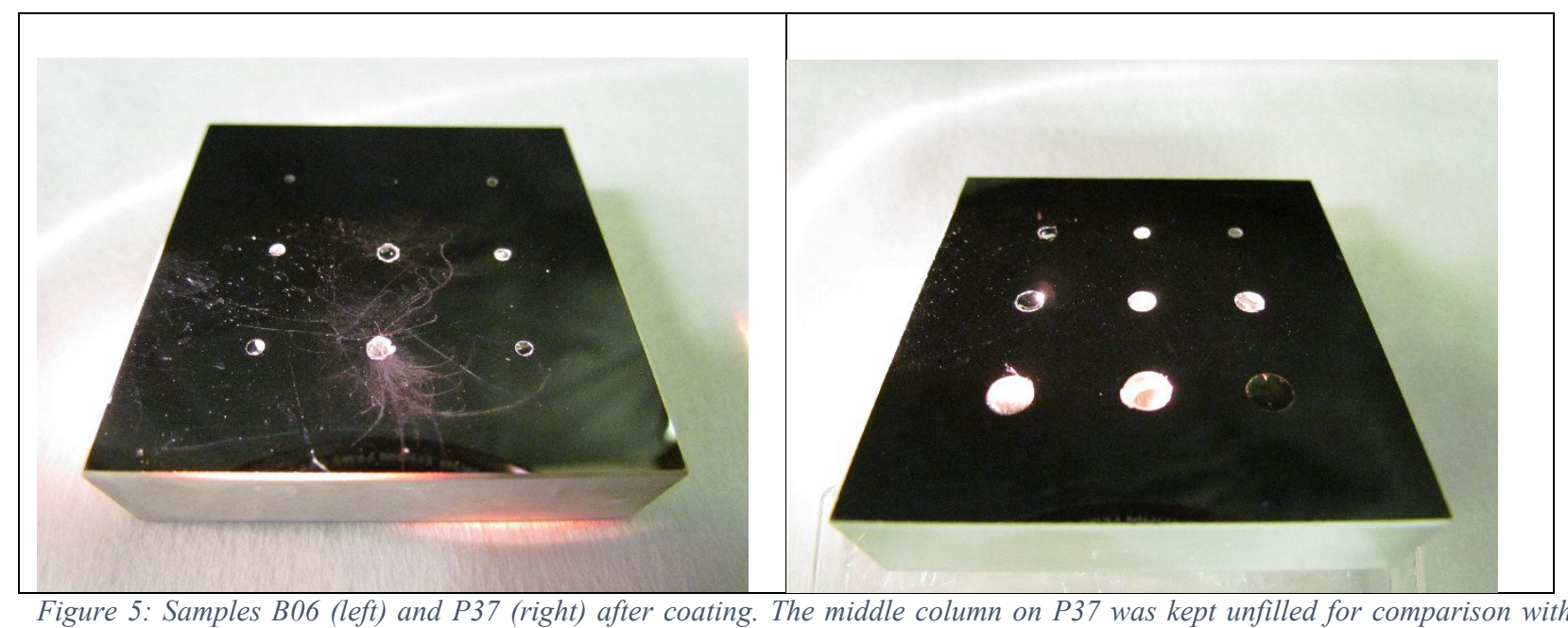
filled holes

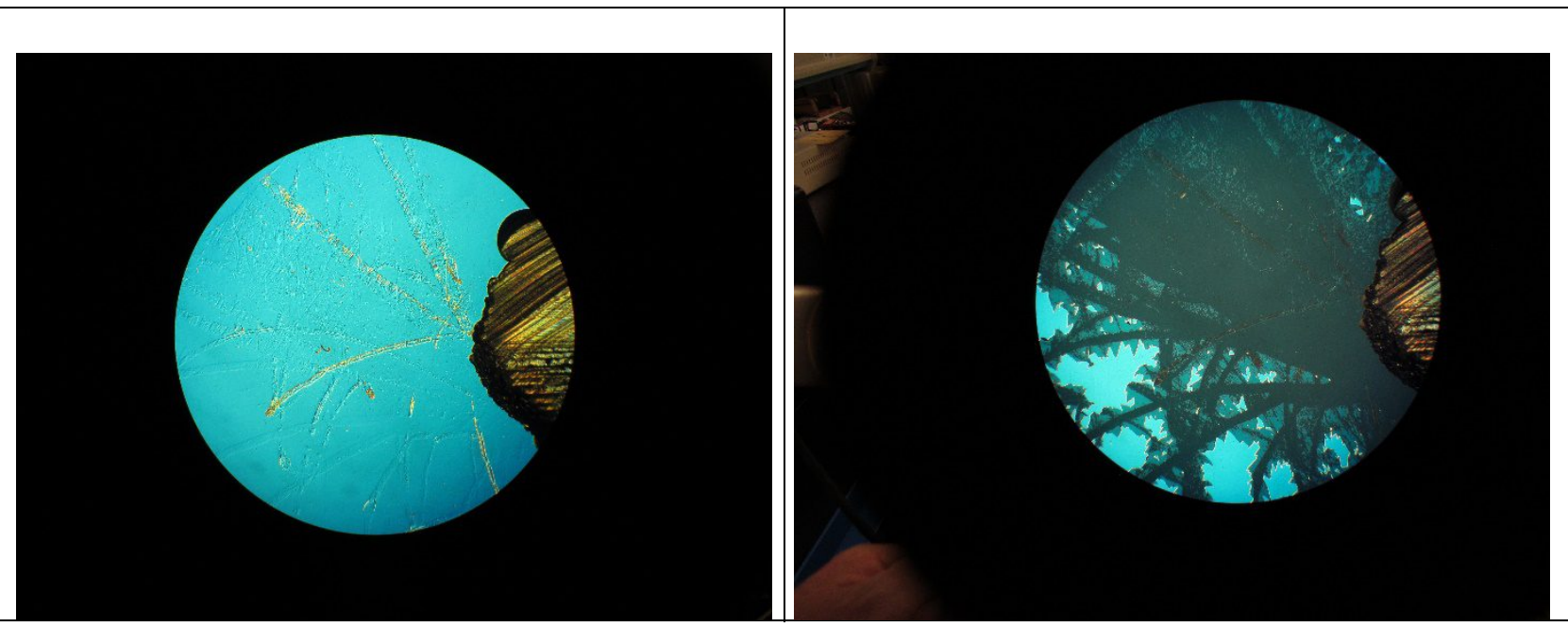

Figure 6: Edge of contaminated hole on B06 before adhesion tape test (left) and after (right)

\section{M1M3 CROW'S FEET}

Crows' feet are narrow trenches extending from open bubbles in the optical surface (figure 7). They are difficult to find by eye on the mirror surface because they are usually small with dimensions not exceeding a depth of $1 \mu \mathrm{m}$, width of a few $\mathrm{mm}$, and length of a few $\mathrm{cm}$. Both M1 and M3 surfaces have such defects, estimated to several hundred altogether. The M3 surface has also larger ones (around two dozen crows' feet) with depths of 2-3 $\mu \mathrm{m}$ and lengths of 20-40 cm. Crows' feet are caused by an interaction between pitch, polishing compound and sharp edges of a hole. Many crows' feet are caused by holes with diameters below $1 \mathrm{~mm}$, thus difficult to identify until a crow's foot develops. Once found, the edge of the hole can be chamfered; the crow's foot stops growing and will shrink with further polishing. 


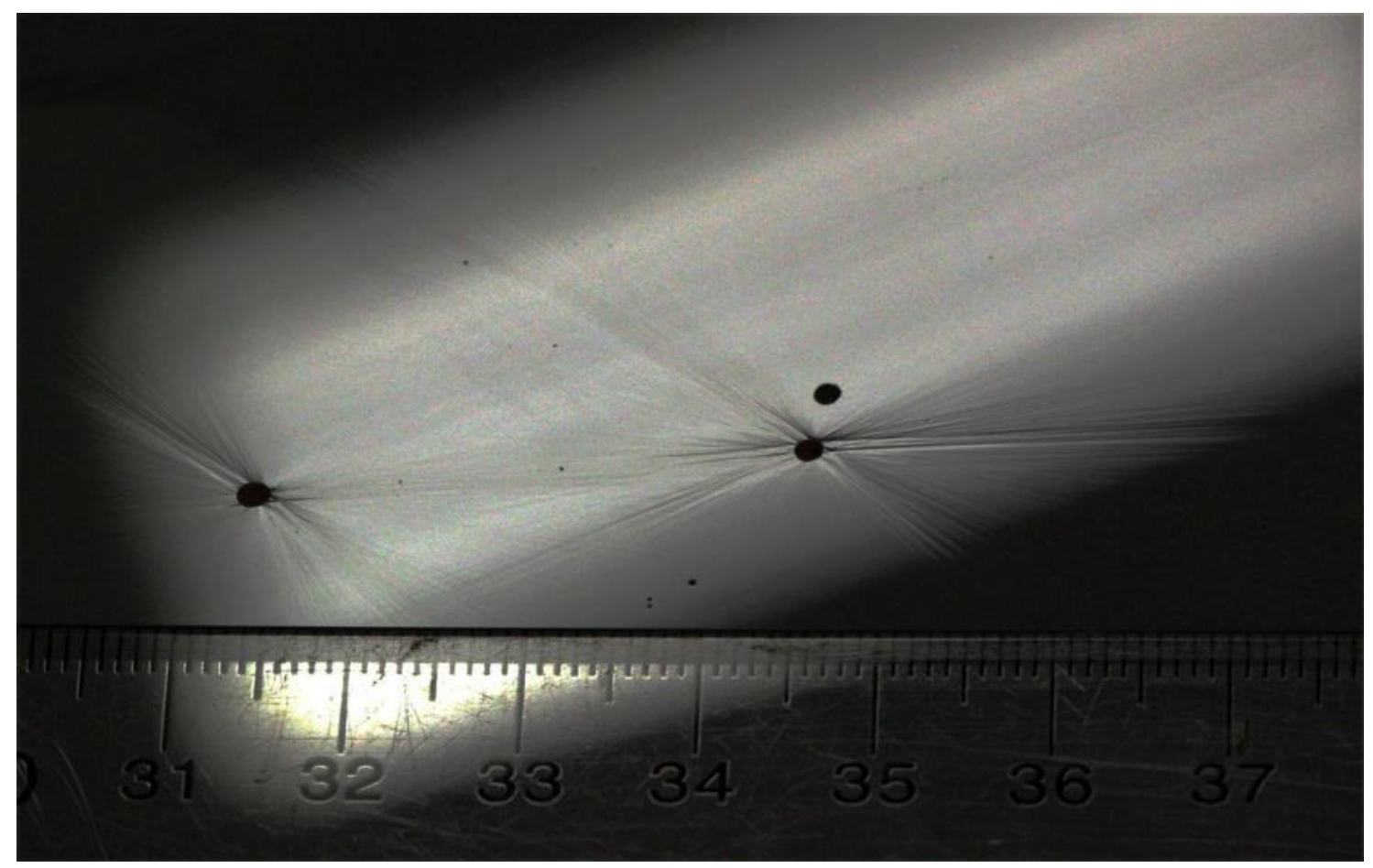

Figure 7: Two of the worst crow's feet on M3, seen as distortion in the reflected image of a fluorescent light. The ruler shows the scale in cm. The two bubbles responsible for the crow's feet have been chamfered to diameters around $1.5 \mathrm{~mm}$.

Removal of crows' feet, or reducing them to a negligible impact, requires the removal of several microns of glass along with smoothing action. This removal and smoothing was achieved for M1 by polishing its surface with the stressed lap. However, due to a stressed lap failure, M3 figuring was finished with smaller tools that are less efficient for bulk removal or smoothing of small-scale structure. Consequently, M3 has a higher density of crows' feet and they are on average longer and deeper than the crows' feet on M1.

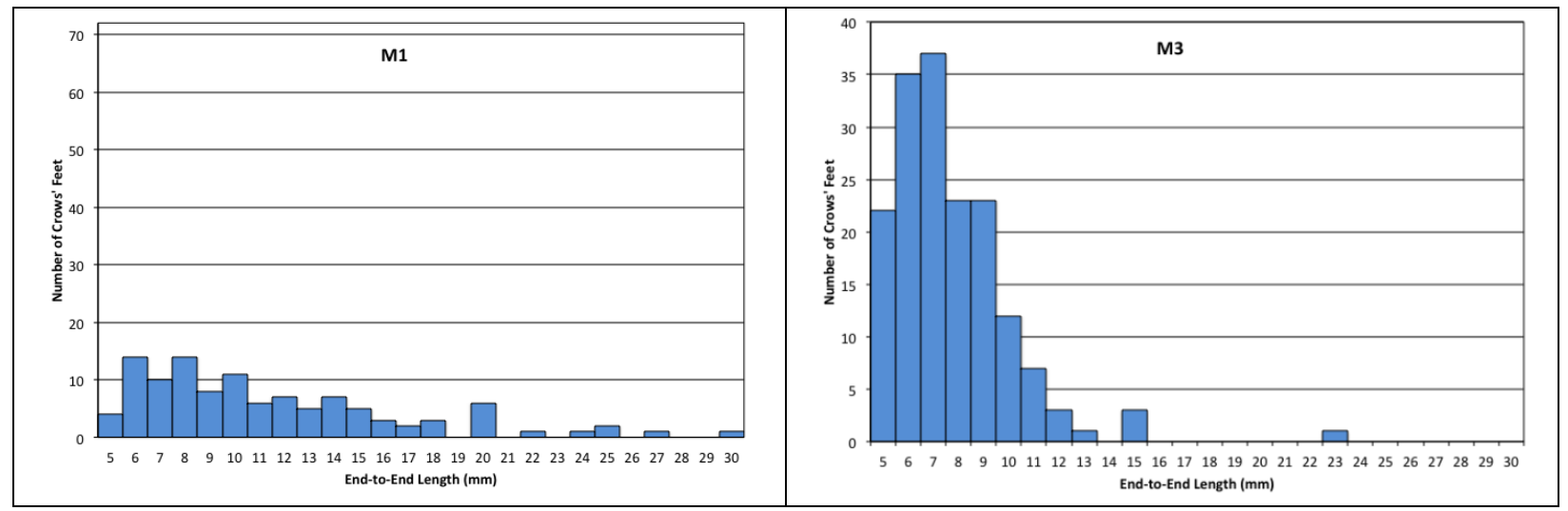

Figure 8: M1 and M3 Crow's feet Histograms of number vs. length, using 1-mm bins for length. The histograms are plotted in a way that makes clear the relative density of crows' feet (number per $\mathrm{m}^{2}$ ) on $M 1$ and M3.

A visual survey was performed and the location, length and width of each crow's foot were recorded down to a length of $5 \mathrm{~mm}$ (figure 8). The vertical axes are plotted so the area in blue on the page is proportional to the density on the mirror surface, taking account of the larger area of M1. The total 
number of crows' feet with length at least $5 \mathrm{~mm}$ is 111 for M1 and 167 for M3. While M1 has 2/3 as many crows' feet as M3, its density of crows' feet is only $37 \%$ that of M3.

The Slope-measuring Portable Optical Test System ${ }^{11}$ (SPOTS) was used to measure the crow's feet as the interferometers' spatial resolution was too low to resolve these defects. A representative survey of about 20 crows' feet on each mirror was used to construct synthetic maps of both optical surfaces with resolved crow's feet features (figure 9). The impact of these features on the LSST point-spread function was determined to be within the image quality error budget ${ }^{8,9}$.

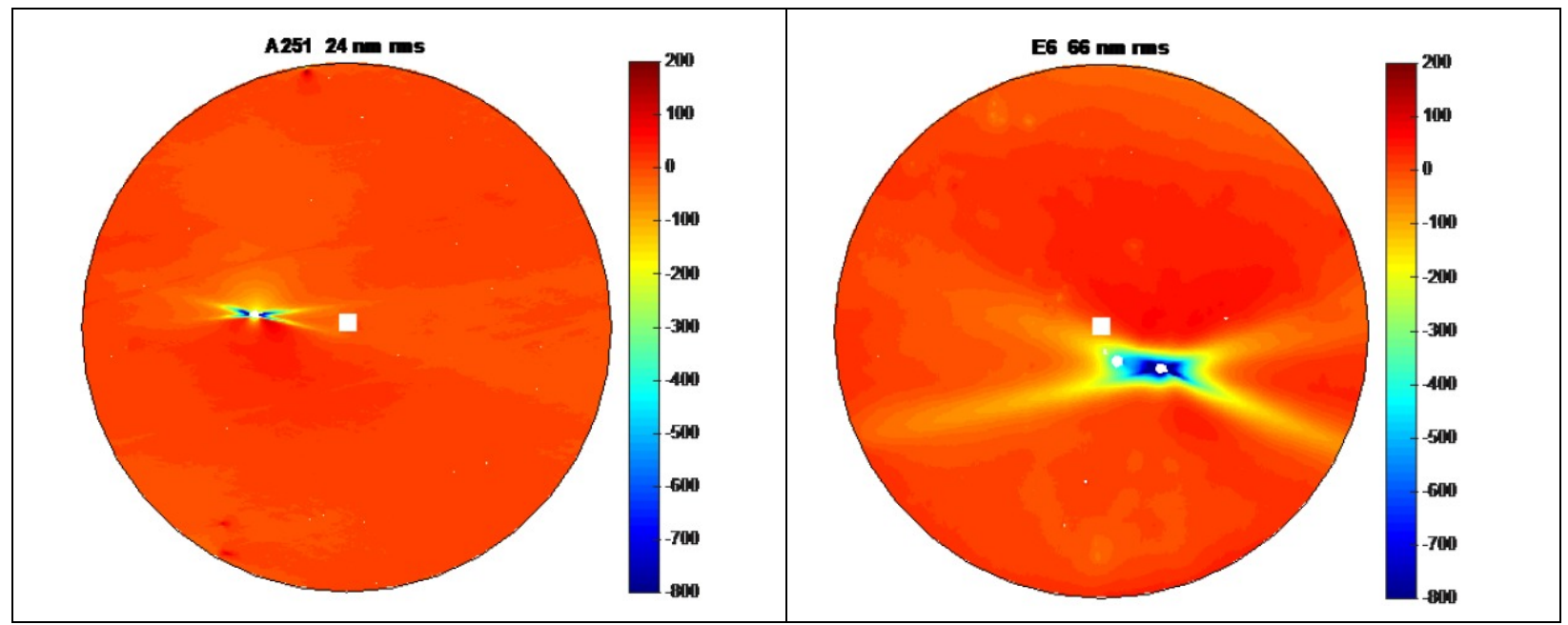

Figure 9: SPOTS maps of crow's foot in M1 (left) and M3 (right), both classified as $15 \mathrm{~mm}$ long in the visual survey. Each map has a diameter of $125 \mathrm{~mm}$. The scale is in $\mathrm{nm}$ (to check)

\section{OPTICAL DESIGN WITH AS-BUILT M1M3}

The impact on the optical design and image quality error budget resulting from the difference between the measured and the required M1M3 mirror performance was estimated. The analysis included optimizing the optical design using the optical design dynamic and static compensators. The dynamic compensators include the 5 degrees of freedom provided by the M2 mirror and the camera hexapods ${ }^{12}$ (rotation around the optical axis is not used). The static compensators include the independent motion along the optical axis of: $a-$ the detector focal plane and b- the assembly composed of [filter, cryostat lens L 3 and detector focal plane].

The optical design was first optimized by updating the radius of curvature, conic constant and vertical separation with the as-built values and optimized by applying the static and dynamic compensators. Then, a second optimization was performed after adding the M3 decenter and M3 wedge but only using the dynamic compensators. As expected, the performance was completely recovered after compensation. The results in terms of encircled energy (EE) averaged over the field of view are presented in the tables below. Averaged $50 \%$ and $80 \%$ EE diameter for all six LSST filters were computed to compare with the performance of the LSST baseline optical design.

The first optimization completely retrieved the baseline performance ${ }^{13}$ (table 3 ) and resulted in using only the motion along the optical axis (piston) to compensate the optical design (table 4). The same analysis 
was repeated using only the dynamic compensators (table 4) and the baseline optical performance was practically retrieved to the same level (table 3 ).

Table 3: 50\% and 80\% Encircled Energy (EE) averaged over the field of view for the six different LSST filters [ugrizY] after the first optimization with static and dynamic compensators (S\&D). All values are in arcsecond.

\begin{tabular}{|c|c|c|c|c|}
\hline $\begin{array}{c}\text { LSST } \\
\text { Filter Band }\end{array}$ & $\begin{array}{c}\text { Averaged EE(50) } \\
\text { with S\&D } \\
\text { compensators }\end{array}$ & $\begin{array}{c}\text { Averaged EE(80) } \\
\text { with S\&D } \\
\text { compensators }\end{array}$ & $\begin{array}{c}\text { Averaged EE(50) } \\
\text { with Dynamic } \\
\text { compensators }\end{array}$ & $\begin{array}{c}\text { Averaged EE(80) } \\
\text { with Dynamic } \\
\text { compensators }\end{array}$ \\
\hline $\mathrm{u}$ & 0.12 & 0.22 & 0.13 & 0.24 \\
\hline $\mathrm{g}$ & 0.12 & 0.23 & 0.13 & 0.23 \\
\hline $\mathrm{r}$ & 0.08 & 0.15 & 0.08 & 0.15 \\
\hline $\mathrm{i}$ & 0.08 & 0.15 & 0.08 & 0.15 \\
\hline $\mathrm{z}$ & 0.08 & 0.15 & 0.08 & 0.15 \\
\hline $\mathrm{Y}$ & 0.08 & 0.15 & 0.08 & 0.15 \\
\hline
\end{tabular}

Table 4: Compensator motions required from the optical design first optimization when using static and dynamic compensators $(S \& D)$ and when using only dynamic compensators. All values are in mm. The positive motion is away from the M1 mirror. The * indicates the value changes a little depending on camera filter.

\begin{tabular}{|l|c|c|}
\hline \multicolumn{1}{|c|}{ Compensator } & $\begin{array}{c}\text { Piston (Z motion) for } \\
\text { S\&D compensators }\end{array}$ & $\begin{array}{c}\text { Piston (Z motion) for } \\
\text { Dynamic compensators }\end{array}$ \\
\hline M2 mirror & 0.141 & 0.002 \\
\hline Camera & $1.298^{*}$ & $0.059^{*}$ \\
\hline Detector Focal Plane & -0.586 & NA \\
\hline Filter + L3 + Detector Focal Plane & -0.281 & NA \\
\hline
\end{tabular}

The second optimization analysis was performed after adding the M3 optical axis decenter and the M3 wedge. The M3 mirror optical axis radial decenter was found equal to $0.4 \mathrm{~mm}$ relative to the M1 optical axis (figure 10). The M3 wedge was computed from the laser tracker measurements repeated three different times. The total indicator run-out (TIR), averaged over these multiple measurements, was found equal to $0.016 \mathrm{~mm}$ with a direction angle of $-100 \mathrm{deg}$.

When added to the optical design, the M3 optical axis decenter and M3 wedge create variations of image quality that is non-symmetrical in the field of view. Consequently, RMS maps of the overall field of view were created to assess the impact of these changes. The $\pm 1.75 \mathrm{deg}$ field was sampled with a $50 \times 50$ position grid and the optimization was performed on all 6 LSST filter bands. 


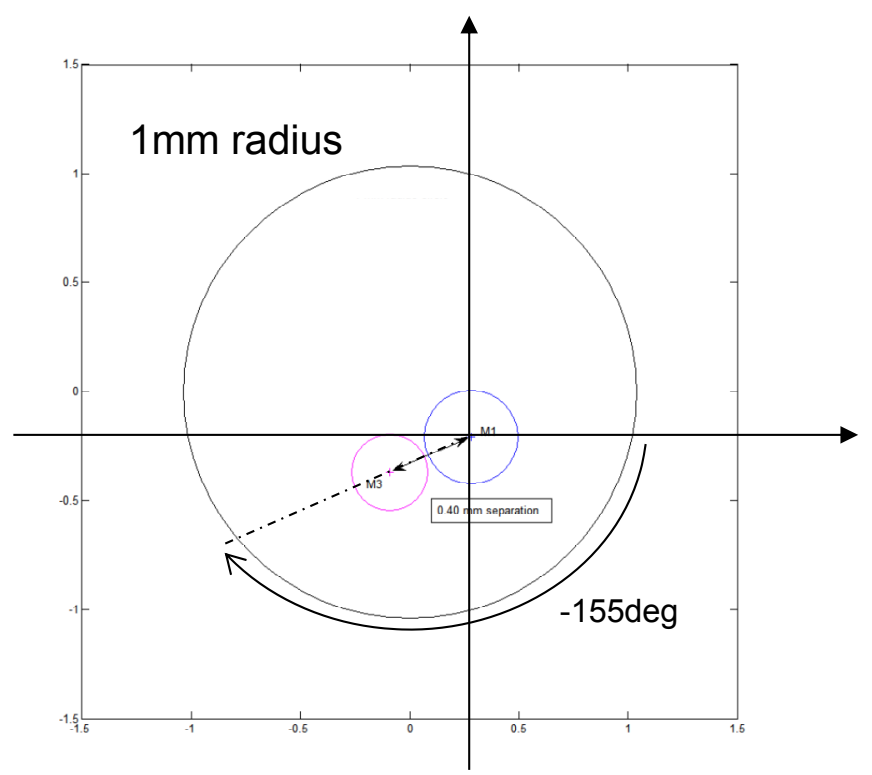

Figure 10: Position of the M3 optical axis relative to the M1 optical axis. Their separation is equal to $0.4 m m$ with a direction angle of -155 degrees. The large circle has a $1 \mathrm{~mm}$ radius and is centered on the M1 mechanical axis. The small circles represent a 2- $\sigma$ uncertainty of $0.3 \mathrm{~mm}$ for $M 1$ and of $0.2 \mathrm{~mm}$ for $M 3$.

Figure 11 shows the RMS field maps for the i-band filter before and after applying the M3 optical axis decenter and M3 wedge without compensators, and also after applying the compensators. The field map starts as circularly symmetric relative to the field center. The RMS spot radius averaged over the field of view is 2.66 microns with a minimum spot radius of 2.46 microns and a maximum of 3.32 microns. After adding the M3 decenter and wedge, the RMS field map is not circularly symmetric anymore and the averaged RMS spot radius becomes 3.64 microns with a minimum spot radius of 2.93 microns and a maximum of 5.37 microns. After compensation, the RMS field map almost completely retrieves its circular symmetry behavior and its original image quality: the averaged RMS spot radius returns to 2.67 microns with a minimum spot radius of 2.44 microns and a maximum of 3.42 microns.

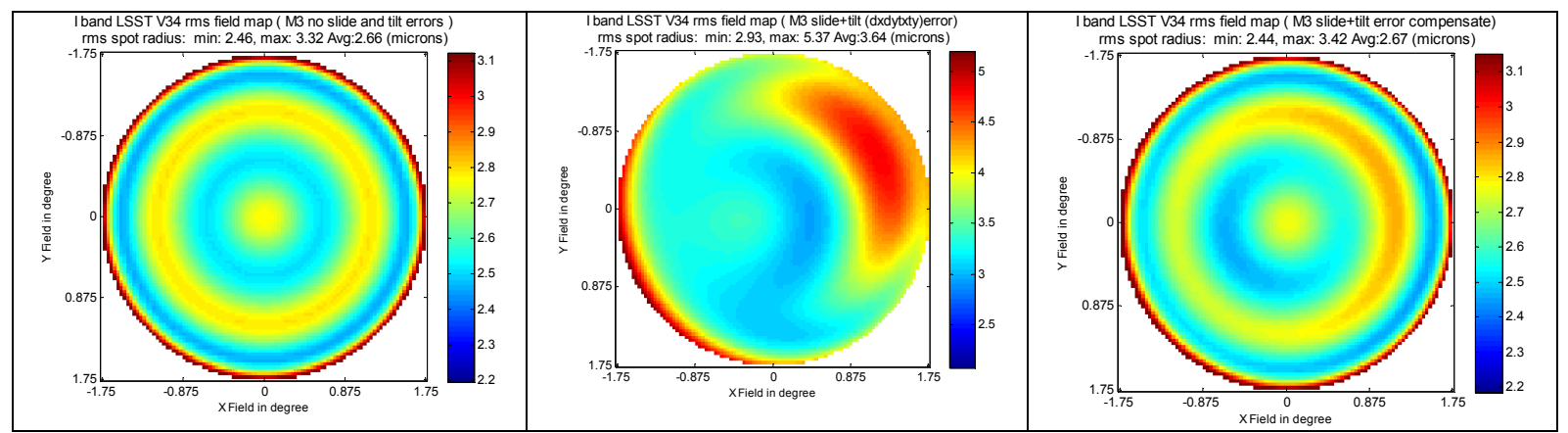

Figure 11: RMS field maps for the i-band filter before (left) and after (center) applying the M3 optical axis decenter and M3 wedge without compensators, and after applying the compensators (right).

The $50 \%$ and $80 \%$ encircled energy diameter were computed along two perpendicular directions (X and Y) to capture the image quality variations within the field of view directions. The results show that it is possible to basically recover the image quality from these fabrication errors by using the dynamic compensators available. Decenter and tilt motions of the M2 mirror and camera were used to compensate the M3 decenter and wedge (table 5). 
Table 5: Decenter and tilt of the M2 mirror and camera to compensate the M3 decenter and wedge

\begin{tabular}{|l|c|c|c|c|}
\hline Compensator & Decenter in X $(\mathrm{mm})$ & $\begin{array}{c}\text { Decenter in Y } \\
(\mathrm{mm})\end{array}$ & $\begin{array}{c}\text { Tilt X } \\
(\operatorname{arcmin})\end{array}$ & $\begin{array}{c}\text { Tilt Y } \\
(\operatorname{arcmin})\end{array}$ \\
\hline M2 mirror & -0.012 & -0.003 & -0.021 & 0.030 \\
\hline Camera & -0.733 & -0.210 & 0.047 & -0.137 \\
\hline
\end{tabular}

\section{M1M3 FINAL FEM}

The LSST team developed a procedure for finalizing the M1M3 finite element model (FEM) using asbuilt data and generating influence matrices for the M1M3 mirror support system. During the process, various types of software such as Matlab, Excel, Visual Studio and NX9 were used.

The M1M3 mirror FEM was updated to implement the drawings produced by RFCML to be consistent with the LSST Telescope coordinate system, to apply the thickness distributions measured on the mirror during fabrication and to match the loadspreader location and numbering. For the correction, the loadspreader numbers were manually re-labelled in Nastran FE NX 9. Then, with the raw thickness variation data measured by RFCML, a Matlab function, scatteredInterpolant, was applied to interpolate the thickness variation in the FEM. Fourteen thickness categories for the faceplate and fifteen for the backplate were created. The interpolated thickness for each element was examined and raised to the closest element category.

In order to validate the final FEM with the previous version, a $1 \mathrm{G}$ axial gravity load was applied along the $\mathrm{Z}$ direction with corresponding pressure in opposite direction to compensate the gravity effect. Then, the lower Zernike terms were removed to validate that the thickness variation of the mirror is conserved. In addition, a $1 \mathrm{G}$ lateral gravity load was applied with the optimum forces defined at 156 axial actuators, 12 $\mathrm{X}$-lateral actuators, and $100 \mathrm{Y}$-lateral actuators. These actuators are part of the M1M3 mirror support system. They support the M1M3 mirror while a set of six hardpoint actuators keeps the M1M3 mirror in position relative to its mirror cell. Less than a $10 \mathrm{~nm}$ RMS surface error was obtained as well as a reasonably well distributed optimum support force sets in $\mathrm{x}, \mathrm{y}$ and $\mathrm{z}$ directions.

A natural frequency analysis was performed with the final FEM (table 6). Results indicated that most frequencies decreased slightly by 1 to 5 percent when compared to the previous model. Influence matrices were created for the M1M3 mirror support control system. The matrices contain 268 unit load cases for 156 axial actuators, $100 \mathrm{Y}$-lateral actuators and $12 \mathrm{X}$-lateral actuators. Active optics performance was demonstrated with sample target displacement sets. The displacement results on the optical surface were post-processed to evaluate the RMS surface error. The best mirror surfaces obtained showed a $10.3 \mathrm{~nm}$ RMS surface error at zenith pointing and a $7.5 \mathrm{~nm}$ RMS surface error at horizon pointing when only zdisplacement is considered for the analysis (figure 12).

Table 6: comparison of the natural frequencies for the first 5 modes between original FEM and final FEM (frequency values are in Hertz).

\begin{tabular}{|l|c|c|c|c|c|}
\hline Modes & 1 & 2 & 3 & 4 & 5 \\
\hline Original FE model & 40.81 & 40.82 & 65.84 & 101 & 101.5 \\
\hline Final FE Model & 39.44 & 39.45 & 63.67 & 97.99 & 98.49 \\
\hline Difference (\%) & 3.36 & 3.36 & 3.30 & 2.98 & 2.97 \\
\hline
\end{tabular}




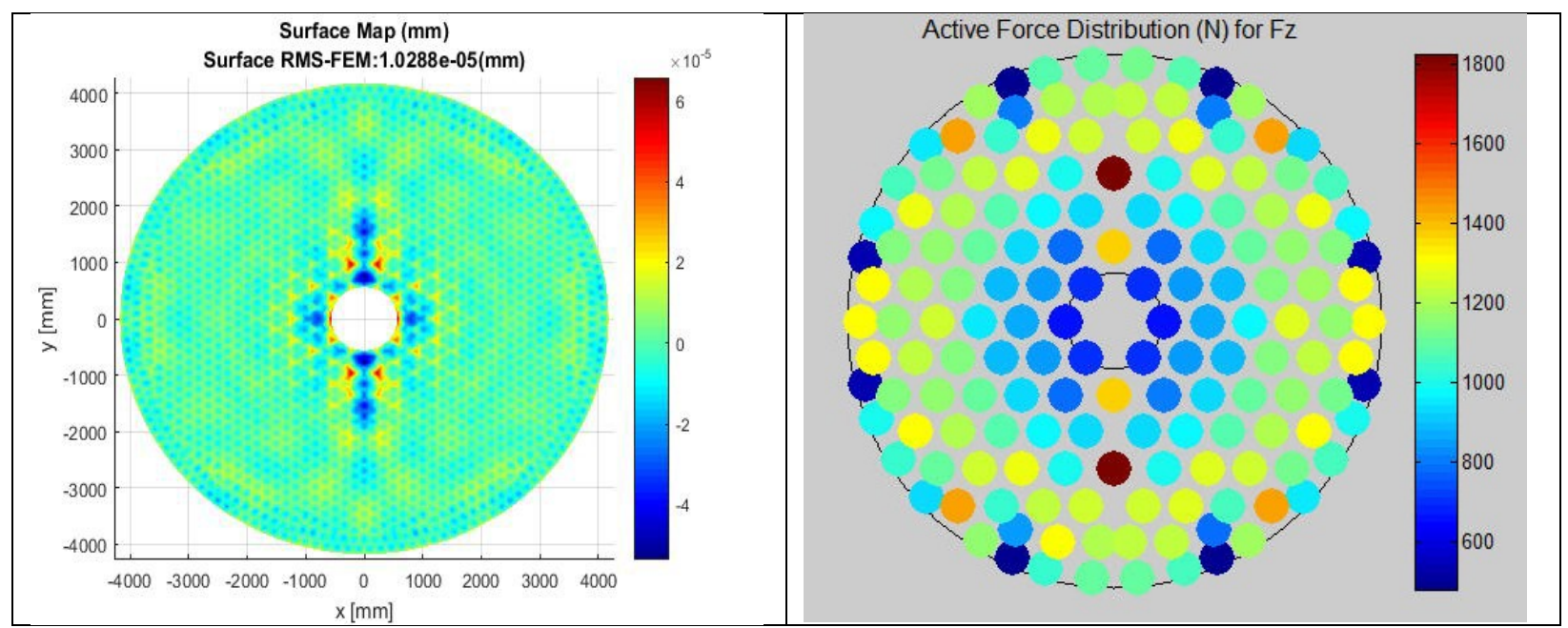

Figure 12: Zenith pointing M1M3 surface RMS error map (left) and zenith pointing optimized mirror support force set distribution

\section{M1M3 MIRROR TRANSPORT TO STORAGE LOCATION}

After final acceptance, the M1M3 mirror was prepared for transfer from its polishing cell into its shipping container. These operations were performed by RFCML personnel including loading of the shipping container on the transport trailer. Precision Heavy Haul (PHH) from Tolleson, AZ was selected to perform the transport of the M1M3 mirror in its container to its storage location in Tucson.

The transportation of the LSST M1M3 mirror in its shipping container began from the receiving area at RFCML. The Shipping Contractor backed the trailer truck into the RFCML loading dock area. RFCML lifted the mirror container using its lifting fixture and lowered it onto the trailer. The Shipping Contractor secured the load on the trailer to exit the loading dock area and begin the transport to Storage.

Early morning on May 19, 2015, pilot cars and police escorts lead the trailer along the route map established during the route survey. Upon arrival at the storage location, PHH placed the shipping container at its reserved space. Finally, the transportation process was completed and the mirror inspected by LSST personnel (figure 13). The mirror will be kept in storage until the mirror cell assembly is ready for a final interferometric test at RFCML with the M1M3 mirror in its final operational cell before shipping to Chile. 


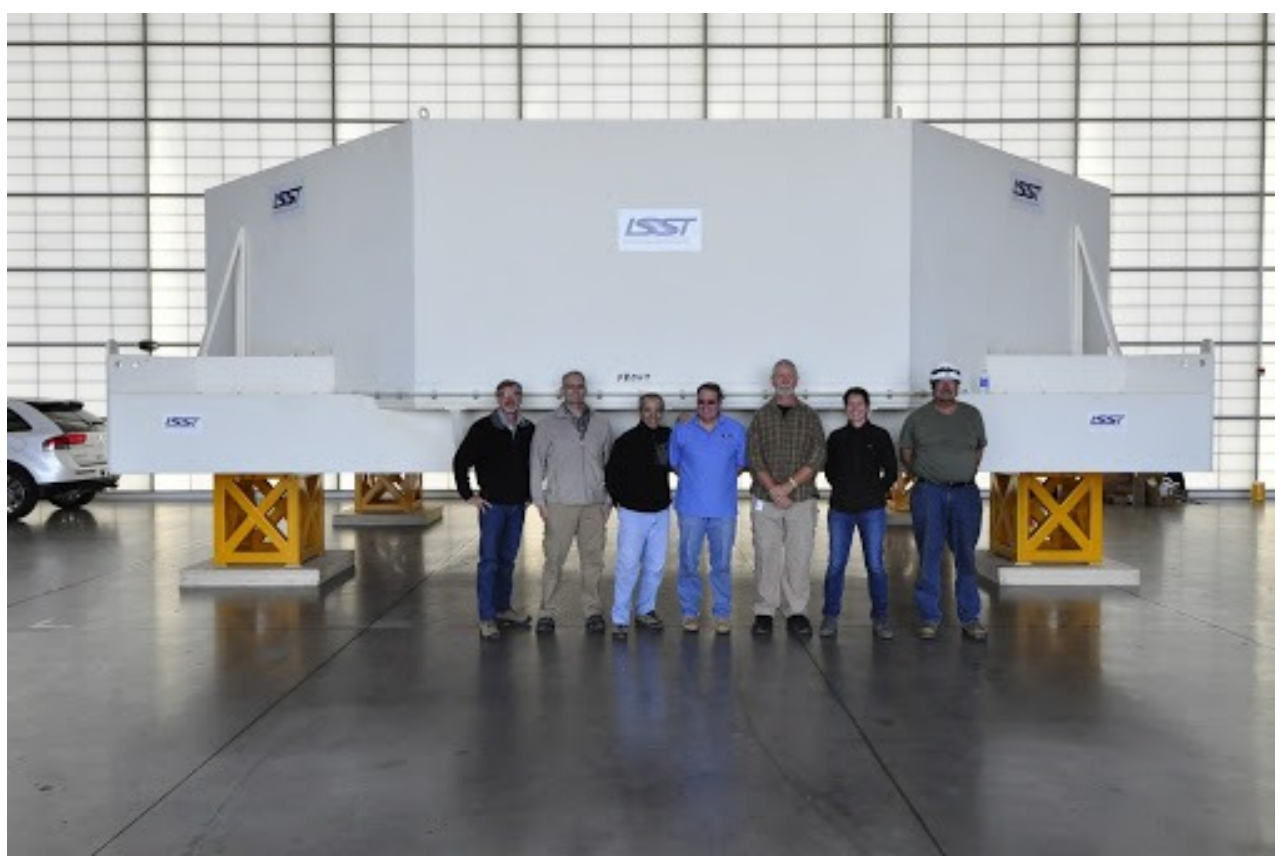

Figure 12: Team picture after storage transportation of M1M3 mirror in its container. From left to right: C. Gessner, B. Gressler, J. Sebag, J. Andrew, G. Poczulp, C. Araujo and R. Repp

\section{CONCLUSION}

The LSST M1M3 mirror, with its uncommon monolithic configuration of 2 large mirrors polished onto one substrate, was successfully delivered within its specifications by RFCML to the LSST project in 2015. It is currently in storage awaiting the fabrication of the M1M3 mirror cell for final testing. Holes and crow's feet affect the mirror surface. It was concluded to not fill the small holes on the M1M3 surface based on the testing done with small samples, and the impact of the crow's feet was estimated acceptable by the LSST project.

\section{ACKNOWLEDGEMENT}

This material is based upon work supported in part by the National Science Foundation through Cooperative Agreement Award No. AST-1227061 under Governing Cooperative Agreement 1258333 managed by the Association of Universities for Research in Astronomy (AURA), and the Department of Energy under Contract No. DEAC02-76SF00515 with the SLAC National Accelerator Laboratory. Additional LSST funding comes from private donations, grants to universities, and in-kind support from LSSTC Institutional Members.

\section{REFERENCES}

[1] Kahn, S., "Final design of the Large Synoptic Survey Telescope," in [Ground-Based and Airborne Telescopes IV], Hall, H. J., Gilmozzi, R., and Marshall, H. K., eds., Proc. SPIE 9906, in press (2016).

[2] Kurita, N., "Large Synoptic Survey Telescope camera design and construction", Proc. SPIE 9912, in press (2016) 
[3] Michael T. Tuell, Hubert M. Martin, James H. Burge, Dean A. Ketelsen, Kevin Law, William J. Gressler, Chunyu Zhao, "Fabrication of the LSST monolithic primary-tertiary mirror", Proc. SPIE 8450, (2012)

[4] Michael T. Tuell, James H. Burge, Brian Cuerden, William Gressler, Hubert M. Martin, Steven C. West, Chunyu Zhao, "Final acceptance testing of the LSST monolithic primary/tertiary mirror," Proc. SPIE 9151 (2014)

[5] Constanza Araujo-Hauck, Jacques Sebag, Ming Liang, Douglas Neill, Sandrine Thomas, Tomislav Vucina, William Gressler, "LSST mirror system status: from design to fabrication and integration," Proc. SPIE 9906 (2016)

[6] Neill, Douglas, R., Muller, G., Hileman, E., Joe, D., Araujo, C., Gressler, W., Lotz, P., Dave, M., Sebag, J., Thomas, S., Warner, M., and Wiecha, O, "Final Design of the LSST primary/Tertiary Mirror Cell Assembly," Proc. SPIE 9906 (2016)

[7] J. Sebag, J. Andrew, G. Angeli, C. Araujo, J. Barr, S. Callahan, M. Cho, C. Claver, F. Daruich, W. Gressler, E. Hileman, M. Liang, G. Muller, D. Neill, B. Schoening, M. Warner, O. Wiecha, B. Xin, Alfredo Orden Martinez, Manuel Perezagua Aguado, Luis García Marchena, Ismael Ruiz de Argandoña, "LSST Telescope Modeling overview," in [Modeling, Systems Engineering, and Project Management for Astronomy VI], Proc. SPIE 9911, in press (2016)

[8] Hubert M. Martin, J. Roger P. Angel, George Z. Angeli, James H. Burge, William J. Gressler, Daewook Kim, Jeffrey S. Kingsley, Kevin Law, Ming Liang, Douglas Neill, Jacques Sebag, Peter A. Strittmatter, Michael T. Tuell, Steve C. West, Neville J. Woolf, Bo Xin, "Manufacture and final tests of the LSST monolithic primary/tertiary mirror," Proc. SPIE 9912 (2016)

[9] G. Z. Angeli, B. Xin, C. Claver, M. Cho, C. Dribusch, D. Neill, J. Peterson, J. Sebag, S. Thomas, "An Integrated Modeling Framework for the Large Synoptic Survey Telescope," in [Modeling, Systems Engineering, and Project Management for Astronomy VI], Proc. SPIE 9911, in press (2016)

[10] J. Sebag, T. Vucina, J. Andrew, D. Neill, G. Poczulp, "Mirror Coatings for the Large Synoptic Survey Telescope: Requirements and Solutions," 59th Annual Technical Conference Proceedings of the Society of Vacuum Coaters, In Press, 2016

[11] Maldonado, A. V., Su, P. and Burge, J. H., "Development of a portable deflectometry system for high spatial resolution surface measurements," Applied Optics, 53, p. 4023-4032 (2014).

[12] Sneed, R., Neill, D., Kidney, S., Araujo, C., Gressler, W., Lotz, P., Dave, M., Sebag, J., Sebring, T., A., Warner, M., and Wiecha, O. "Final design of the LSST hexapods and rotator," Proc. SPIE 9906 (2016).

[13] Seppala, L. G., "Improved optical design for the Large Synoptic Survey Telescope (LSST)," Proc. SPIE 4836 (2002) 